\section{PROMOCIÓN DE LA JUSTICIA FRENTE A “UTILITARISMO” EN EL PENSAMIENTO UNIVERSITARIO DEL SUPERIOR GENERAL DE LA COMPAÑÍA DE JESÚS PETER HANS KOLVENBACH}

\author{
Borja Vivanco Díaz \\ Infyde \\ borjavivanco@infyde.eu
}

Cómo citar este artículo/Citation: Vivanco Díaz, B. (2016). Promoción de la justicia frente a "utilitarismo" en el pensamiento universitario del superior general de la Compañía de Jesús Peter Hans Kolvenbach. Arbor, 192 (782): a357. doi: http://dx.doi. org/10.3989/arbor.2016.782n6002

Recibido: 04 noviembre 2014. Aceptado: 25 agosto 2015.

RESUMEN: El objetivo de este artículo consiste en dialogar con los discursos universitarios de Peter Hans Kolvenbach, superior general de la Compañía de Jesús entre 1983 y 2008. En particular analizamos la dimensión "iustitia" como elemento distintivo del paradigma de la educación superior jesuita y todo ello lo contextualizamos, a la vez, en su tradición histórica. La apuesta por la promoción de la justicia social, en el entramado universitario de la orden de Ignacio de Loyola, responde asimismo a las opciones que la Compañía de Jesús ha tomado, a raíz del Concilio Vaticano II, y se posiciona como una alternativa a la óptica utilitarista.

PALABRAS CLAVE: Compañía de Jesús; Iglesia Católica; Ignacio de Loyola; Justicia; Paradigma; Universidad; Kolvenbach.

\section{PROMOTION OF JUSTICE VERSUS "UTILITARISM" IN THE SCHOLARLY THINKING OF THE SUPERIOR GENERAL OF THE SOCIETY OF JESUS, PETER HANS KOLVENBACH}

Copyright: (C) 2016 CSIC. Este es un artículo de acceso abierto distribuido bajo los términos de la licencia Creative Commons Attribution (CC BY) España 3.0.
ABSTRACT: This paper addresses the discourse in relation to universities started by Peter Hans Kolvenbach, Superior General of the Society of Jesus between 1983 and 2008. In particular, we analyze the "iustitia" dimension as a distinctive element in Jesuit higher education and contextualize it within its historical tradition. The bid to promote social justice, in the university framework of the order of Ignacio of Loyola, also conforms with the standpoint that the Society of Jesus has had since the Second Vatican Council, positioned as an alternative to the utilitarian perspective.

KEYWORDS: Society of Jesus; Catholic Church; Ignacio of Loyola; justice; paradigm; university; Kolvenbach. 


\section{INTRODUCCIÓN}

Aunque germinó en la Universidad de París, ${ }^{1}$ la Compañía de Jesús no tuvo intención, en un primer momento, de dedicarse a la enseñanza, tampoco a la educación superior. ${ }^{2}$ Los jesuitas sabían de otros clérigos como los dominicos, que desde siglos atrás se dedicaban al mundo universitario, pero no les atraía su estilo de vida particular. Dicho lo cual, la decisión de incorporarse a los apostolados educativo e intelectual supuso una especie de "terremoto" en la primera generación de jesuitas. " "Sentían que se les movía el suelo", llegaron a confesar algunos de ellos (Sánchez Marco, 2010, p. 455).

Pero a la muerte de Ignacio de Loyola, acaecida en 1556, 35 de los 40 colegios que él había aprobado -en persona- continuaban funcionando. Mientras que cuatro décadas más tarde el número de colegios ascendía ya a 245 (Características de la educación de la Compañía de Jesús, p. 51).

La primera universidad jesuita fue constituida en 1547, en la ciudad de Gandía. Desapareció, para siempre, al poco de ser expulsados los jesuitas de España en 1767, por orden del rey Carlos III. Fue fundada por Francisco de Borja, quien llegó a ser tercer superior general de la Compañía de Jesús. Más tarde, en 1551, el mismo Ignacio de Loyola creó el Colegio Romano, germen de la emblemática Universidad Gregoriana, localizada en el corazón de la ciudad del Tiber. Ignacio de Loyola (Plazaola, 1999, p. 15) tuvo intención de que el Colegio Romano fuera modelo para todos los demás que más tarde se fundarían.

En 1975 la Congregación General XXXII liderada por el superior general Pedro Arrupe, y convocada para clarificar y dar el espaldarazo a la renovación de la Compañía de Jesús inspirada en el Concilio Vaticano II, ${ }^{4}$ reubicó -inéditamente en su historia- la misión de la Compañía de Jesús en términos de propagación de la fe y promoción de la justicia, como si de una misma labor se tratase. ${ }^{5}$

Sobre todo el Decreto 4 de la Congregación General XXXII recogió las nuevas orientaciones de la Compañía de Jesús. Aunque el Decreto 4 priorizó la justicia social $^{6}$ en las tareas de apostolado de los jesuitas, no ignoró -ni mucho menos- otros valores cristianos. Más bien, el Decreto 4 deseaba aprovecharlos para la causa de la justicia social y, favoreciéndola, era su deseo también enriquecerlos. ${ }^{7}$

En cualquier caso debemos incidir en que, desde el punto de vista de la evolución de la Compañía de
Jesús, el Decreto 4 es un hito fundamental, que contribuye a superar plenamente el concepto de beneficencia o caridad limosnera concediendo a la lucha por la justicia y por el cambio estructural un lugar prioritario. María Jesús Cava Mesa (2007, p. 222) lo ha explicado con estas palabras:

"En la Iglesia de Occidente la fe y la justicia habían estado, si no divorciadas -y cada una en busca de otro casamiento-, al menos muy separadas. En vez de la justicia se iba por el camino de la caridad limosnera. Arrupe y su congregación quisieron dar un vuelco decisivo a esta situación verdaderamente escandalosa para la fe".

Por otro lado es indudable que el futuro de los grupos sociales más desfavorecidos se juega también en el tablero académico e intelectual. ${ }^{8}$ Más exactamente, puede proclamarse que la institución universitaria está llamada a ocupar un lugar insustituible ${ }^{9}$ en la defensa de los colectivos sociales vulnerables ${ }^{10} \mathrm{y}$, principalmente, en una triple dirección:

- Para denunciar injusticias y debatir las vías más adecuadas para solventarlas. ${ }^{11}$

- Para formar a minorías sensibles a sus derechos y necesidades.

- Para investigar las causas, las características y las consecuencias de las situaciones de desigualdad.

A lo largo de las páginas siguientes ${ }^{12}$ vamos a llevar a cabo un estudio de los discursos universitarios del superior general de la Compañía de Jesús Peter Hans Kolvenbach, analizando especialmente aquellos elementos más vinculados a la promoción de la justicia social. ${ }^{13}$

\section{CARACTERÍSTICAS DEL PARADIGMA DE LEDESMA - KOLVENBACH}

El modelo de Ledesma - Kolvenbach se conoce como el paradigma de la misión educativa de los centros de estudios superiores de la Compañía de Jesús, formulado por quien fue máximo responsable de la orden -Peter Hans Kolvenbach- ${ }^{14}$ entre 1983 y 2008, a partir de sobre todo sus últimos discursos pronunciados en las universidades jesuitas. En otros establecimientos educativos de los jesuitas (colegios, centros de Formación Profesional,...), no se ha diseñado ningún otro paradigma de este estilo o con este tipo de definición. ${ }^{15}$

El paradigma de Ledesma - Kolvenbach ha sido bien recibido en el mundo universitario jesuita y está siendo tomado como referencia, para orientar la misión, la gestión y la actividad de los centros de estudios su- 
periores. Son muchos los desafíos y los cambios tan rápidos que se han ido operando, dentro y fuera de las universidades jesuitas, y que han exigido una clarificación de su identidad y misión. ${ }^{16}$ Nos referimos a cuestiones tales como:

- La instauración de la educación para la justicia en el corazón de la cultura de enseñanza de la Compañía de Jesús.

- La preponderancia del "utilitarismo" en la praxis universitaria. ${ }^{17}$

- Las dimensiones tan colosales que muchas de estas universidades han ido alcanzando en los últimos tiempos.

- El incremento de la colaboración y de la implicación activa de los laicos en estas universidades.

- El número de jesuitas tan insignificante que trabajan en algunas de las más emblemáticas y la incorporación de laicos en sus aparatos de gestión.

- Las nuevas directrices educativas establecidas desde instancias públicas y que reclaman también su cumplimiento (Declaración de Bolonia, por ejemplo) por parte de las universidades no civiles,...

- La irrupción de la Responsabilidad Social Universitaria (RSU) como modelo teórico. ${ }^{18}$

Por supuesto, el modelo que estamos estudiando tiene más que ver con el pensamiento de Kolvenbach que con Diego Ledesma y sus disquisiciones pedagógicas, que de manera tan notable contribuyeron a la elaboración de la Ratio Studiorum de 1599; que fue el primer plan de estudios para los colegios y las universidades de la Compañía de Jesús. ${ }^{19}$ Sin embargo, acudiendo a Ledesma, Kolvenbach buscaba también inspirarse en la primigenia pedagogía jesuita y en quienes, antes que él, se han sentido herederos de ella, a lo largo de más de cuatro siglos de historia. Todo esto permite igualmente que el paradigma no se llegue a representar en absoluto como ruptura; sino como una evolución natural de los apostolados educativo e intelectual de la Compañía de Jesús, adaptados a los nuevos tiempos y a las necesidades de la Iglesia Católica y de la Humanidad.

La dimisión de Kolvenbach en 2008, como superior general de la Compañía de Jesús, no ha disminuido la importancia de este paradigma en el seno de las universidades jesuitas. Su sucesor, el español Adolfo Nicolás que presidió la orden desde 2008 hasta 2016, lo tomó como propio; según fue demostrando en sus discursos universitarios. Previsiblemente Arturo Sosa, elegido sucesor de Ignacio de Loyola en octubre de 2016, durante la Congregación General XXXVI, profundizará en el paradigma. Sosa conoce, de manera muy cercana, el mundo universitario jesuita. De hecho, ha sido rector de la Universidad Católica del Táchira, en Venezuela.

Es bien conocido que son cuatro los conceptos que definen al modelo de Ledesma - Kolvenbach y que, a su vez, buscan sintetizar la identidad y la misión de las universidades jesuitas, en cualquier lugar del planeta: "Utilitas", "iustitia", "humanitas" y "fides". ${ }^{20}$

La diferenciación de cuatro dimensiones no debe apartarnos de la idea de que estamos ante un único modelo. En palabras de Melecio Agúndez (2008, pp. 605-606), uno de los jesuitas que más y mejor han analizado el paradigma de Ledesma - Kolvenbach, además de haber sido quien acuñó su denominación, "la distinción de cuatro dimensiones es un artificio válido para el análisis, pero podrían perturbar la verdadera comprensión del todo, que es unidad dinámica, síntesis interdependiente". Seguidamente, Agúndez contemplaba (en este paradigma) "dos dinámicas interdependientes":

- Interdependencia de las cuatro dimensiones (unidad del modelo). Son "cuatro aspas" de una "hélice" que gira al ritmo de un solo "eje", que son los valores "I+M" (Identidad y Misión). Lo que viene a decir que "en las finalidades pedagógicas de la enseñanza jesuítica, no es comprensible la utilitas (...) sin la iustitia (...); ni aquella y ésta sin la humanitas, (...) y ninguna de las tres sin la fides". O, de modo invertido, podría también afirmarse que "la dimensión radical de sentido alimenta, moviliza y potencia el soporte humano que, con responsabilidad social, desarrolla su competencia profesional útil en provecho propio y de la sociedad".

- Interrelación del contexto y los fines pedagógicos (unidad del sistema): Las cuatro variables se ubican "en un espacio limitado por los planos institucional, curricular, extra-curricular, de investigación...". Es decir, "el sistema institucional influye y es influido por el funcionamiento del Modelo cuatridimensional".

En opinión de Kolvenbach, estos cuatro principios argumentaban la presencia de los jesuitas en el campo universitario, ya a la mitad del siglo XVI. Por esta razón, Kolvenbach (2008, pp. 258-259) hacía suyas las palabras que Ledesma escribió, a los pocos años de 
fundarse la Compañía de Jesús, y referidas a los objetivos de las universidades jesuitas:

“Lo primero, porque proveen a la gente con muchas ventajas para la vida práctica; en segundo lugar, porque contribuyen al correcto gobierno de asuntos públicos y a la apropiada formulación de leyes; en tercer lugar, porque dan decoro, esplendor y perfección a nuestra naturaleza racional; y en cuarto lugar, que es de suma importancia, porque son la defensa de la religión y nos guían con gran seguridad y facilidad en la consecución de nuestro fin último".

\section{“UTILITAS” VERSUS “UTILITARISMO”}

La primera dimensión incluida en el paradigma de Ledesma - Kolvenbach es la "utilitas", que viene a subrayar el carácter práctico de la misión y la enseñanza universitaria. Es también, como sabemos, una variable inherente a cualquier proyecto educativo de carácter universitario del siglo XXI. Pero el cultivar la actitud y el aprendizaje prácticos ha sido asimismo un rasgo distintivo de la espiritualidad ignaciana o de la pedagogía jesuita; en la meta de adecuar los medios a los fines, de facilitar la inserción o la adaptación al mundo real.

El proporcionar una formación total dirigida a solventar las necesidades futuras de los estudiantes, y a lo largo de toda su vida, ha supuesto un elemento tan central como sustantivo en los objetivos pedagógicos de los colegios y las universidades jesuitas. ${ }^{21}$ Por ejemplo en 2006, en su discurso a las facultades universitarias de Notre Dame de la Paix, Peter Hans Kolvenbach (2008, p. 251) se expresaba de esta manera:

Cfr. 7 "La educación jesuítica quiere ser eminentemente práctica, con la mira puesta en asegurar a los estudiantes y a las estudiantes los conocimientos y las competencias con las que podrán sobresalir en el campo de especialización que hubieren elegido".

Es aceptable que haya universidades que privilegien la "utilitas" frente a cualquier otra dimensión. Ahora bien, creemos que el concepto de "utilitas" en el paradigma de Ledesma - Kolvenbach se contrapone extraordinariamente, en su significado y en su función, al "utilitarismo"; en la medida en que se complementa y se interrelaciona, al mismo nivel, con las otras tres dimensiones.

En 2007, durante la conferencia que pronunció en la Universidad Gregoriana de Roma, Kolvenbach (2008, p. 260) arremetía contra la práctica del "utilitarismo" en el mundo universitario:
Cfr. 8 "Concentrarse exclusivamente en los elementos pragmáticos de la educación, sólo en el progreso económico, simplemente en el progreso científico y tecnológico, solamente en intereses económicos, puede fácilmente reducir el fin práctico de una Universidad a una estrecha perspectiva que convierte las otras tres metas de la vida universitaria en meras abstracciones. Más bien, una Universidad jesuita será eminentemente práctica cuando siga insistiendo en una formación integral y un enfoque holístico de la educación".

La finalidad de las universidades jesuitas no puede limitarse nunca a la "utilitas", aunque no pocas hayan podido pecar de ello en algunos momentos. Porque, si esto ocurriera, la "utilitas" se transformaría en "utilitarismo"; corrompiendo la esencia del sistema universitario y de su mejor tradición. El "utilitarismo" podría convertir a la universidad en una institución sin valores y ningunearía su potencial contribución como bien social.

No obstante, es conocido por todos que un buen número de universidades públicas y privadas restringen, totalmente o casi, su proyecto educativo a la variable "utilitas" y que, además, lo han formulado así sin complejos. Optan por interpretar la dimensión "utilitas" como el proporcionar, al alumnado, la base y la cualificación necesarias -y fundamentalmente técnicas- para que pueda insertarse en el mercado de trabajo de manera inmediata y a través de una ocupación con las mejores condiciones laborales; pensando que así conseguiría su pleno "desarrollo profesional". Hacia esta dirección, las "universidades utilitaristas" focalizan la totalidad de sus recursos, propósitos e iniciativas. Y, en este contexto, el éxito y el desarrollo profesional se miden en función de la posibilidad para promocionar laboralmente cuanto antes y desempeñar puestos de trabajo cada vez mejor remunerados; ignorando casi siempre la responsabilidad social y la ética. Explícita o implícitamente, el modelo de "universidad utilitarista" deja en manos del mercado -casi de manera exclusiva- la definición del perfil competencial del joven titulado. ${ }^{22}$

En 2011 Nicolás (Lección inaugural del curso académico 2011-2012 en la Universidad de Deusto, p. 3), en la Universidad de Deusto, denunciaba este modelo de sistema universitario, del que era consciente que no paraba de seguir expandiéndose en nuestros días:

"En esta deriva, la universidad, de ser concebida como lugar de búsqueda del conocimiento camina hacia una universidad casi en exclusiva profesionalizante. El conocimiento ha dejado de ser un fin en sí 
mismo y se ha convertido en una mercancía susceptible de ser vendida y comprada. Esto provoca la consiguiente desvalorización de las disciplinas que tienen poco que ofrecer al mundo comercial".

Y el mismo Kolvenbach (2008, p. 203) reconocía que la visión "utilitarista" podía también absorber la praxis de la actividad universitaria de la Compañía de Jesús, adulterando sus mejores ideales. En Roma, en el año 2001, ante representantes de centros de estudios superiores de la Compañía de Jesús, hablaba de esta manera:

Cfr. 36 "No nos engañemos: cuántos de nuestros estudiantes acuden a nuestras universidades simplemente en búsqueda de la excelencia que ofertamos, y de una capacitación que les permita conseguir un buen puesto de trabajo y mejorar sus ingresos. Algunos pueden pasar años en nuestras instituciones de educación superior, sin enterarse siquiera que se trata de una institución católica dirigida por la Compañía de Jesús".

Desde luego, no ha preocupado solo a los jesuitas que la universidad pueda caer en el "utilitarismo". Es, por ejemplo, un asunto al que inicialmente el conjunto de las universidades católicas buscan contraponerse a toda costa. En 2011, en su discurso ante representantes del entorno universitario, pronunciado en el monasterio de San Lorenzo de El Escorial, cerca de Madrid, Benedicto XVI (2011, p. 5) advertía también, y de manera muy crítica, de los riesgos de que la universidad sucumbiera al "utilitarismo"; que podría conducirla a "los abusos de una ciencia sin limites" y al "totalitarismo político que se aviva fácilmente cuando se elimina toda referencia superior al mero cálculo de poder".

"A veces se piensa que la misión de un profesor universitario sea hoy exclusivamente la de formar profesionales competentes y eficaces que satisfagan la demanda laboral en cada preciso momento. También se dice que lo único que se debe privilegiar en la presente coyuntura es la mera capacitación técnica. Ciertamente, cunde en la actualidad esa visión utilitarista de la educación, también la universitaria, difundida especialmente desde ámbitos extrauniversitarios".

\section{DEL “UTILITARISMO” A LA “UTILITAS”}

La "utilitas" puede contribuir a que la sociedad civil obtenga altas cuotas de "iustitia", mayormente en la medida que facilita la empleabilidad y es motor para el crecimiento económico y el progreso tecnológico y científico; que a su vez son potenciales fuentes de todo tipo de desarrollo cultural y, por supuesto, social. Pero muchas veces ocurre que la dimensión "utilitas", al mutar en "utilitarismo", no mitiga las desigualda- des sociales, puede ahondar más en ellas y requiere, en consecuencia, ser modelada por otras; como por ejemplo las variables "humanitas" y "iustitia" que forman parte del modelo de Ledesma - Kolvenbach.

Hay un componente ético que distancia, de modo definitivo, al "utilitarismo" de la "utilitas". Según el jesuita Augusto Hortal Alonso (2008, pp. 636-637), "para el utilitarismo no hay acciones que sean buenas o malas en sí; el único criterio de moralidad se toma del cálculo de las consecuencias que dicha acción reporta para la mayor felicidad del mayor número de personas afectadas por dicha acción". Y esta "felicidad" se puede traducir en "todo tipo de placer o ausencia de dolor". Mientras que la "utilitas" no es únicamente "para disfrutar, sino para vivir y ayudar a vivir con dignidad y en plenitud, para una mejor humanitas, una mayor iustitia, una mejor fides, es decir para mejor amar y servir en todo", según una simbiosis que hace el citado autor del paradigma de Ledesma - Kolvenbach y las palabras de Ignacio de Loyola.

El contribuir a la inserción laboral de un joven (preferentemente en situación o riesgo de exclusión social) supone ya, en sí mismo, cumplir una finalidad social de primer orden y que no costaría ser también descifrada en términos de justicia social.

Pero el impacto sobre la justicia social de un joven recién titulado será mucho mayor si, además, conoce y está sensibilizado con los problemas y las aspiraciones de los colectivos más desfavorecidos; si desarrolla su actividad profesional en un ámbito en el que puede influir especialmente en ello; si está dispuesto también a colaborar (en su tiempo de ocio) en esta misma causa y si, por supuesto, ha adquirido en la universidad los valores y las competencias necesarias en este menester. Es más, un joven que acaba de finalizar sus estudios y que accede al mercado laboral, si actúa con "neutralidad" en su desempeño profesional y en su vida cotidiana o si las organizaciones (empresa, partido político,...) en las que pudiera participar también lo hacen, no tardaría en ser cómplice o hasta podría ser tachado de culpable por cometer injusticias sociales.

Para que los centros de estudios superiores, jesuitas o no, promuevan el primer perfil de titulado requieren impulsar proyectos educativos, en los que los objetivos y los contenidos de las dimensiones "humanitas" y "iustitia" estén presentes en un lugar prioritario. En el viaje que las universidades emprendan, para regresar del "utilitarismo" a la "utilitas", es oportuno transcurrir por los senderos abiertos por ambas variables. 
Es decir, el fomentar la empleabilidad de los jóvenes debe evitar, a toda costa, la cultura del "sálvese quien pueda" y sí, muy al contrario, ser capaz de facilitar la asimilación de valores solidarios o socialmente responsables.

La perspectiva "fides" no tiene que ser, en efecto, imprescindible. No se pone en duda que un $\mathrm{Hu}$ manismo laico pueda igualmente ser propulsor del compromiso entero, eficaz y definitivo por la justicia social. Sin embargo es sólido que la "fides", además tal y como está formulada en el paradigma de Ledesma - Kolvenbach y en los decretos de la Compañía de Jesús desde la Congregación General XXXII, es impulsora de alternativas centradas en la lucha por la justicia social.

\section{LA “HUMANITAS” EN LA TRADICIÓN JESUITA A FAVOR DEL HUMANISMO Y DE LA FORMACIÓN INTELECTUAL Y RACIONAL}

No cabe duda que el cultivo de las humanidades ha conformado una de las grandes aportaciones y también una de las señas de identidad de las universidades jesuitas. La Compañía de Jesús, junto a sus colegios y a sus universidades, emergió de modo simultáneo al Humanismo. ${ }^{23}$ Cuando hablamos del Humanismo nos referimos a la consolidación del antropocentrismo y al punto final de la sociedad teocrática, al surgimiento del arte renacentista y a la recuperación de la cultura clásica, heredada de las civilizaciones griega y romana. Las universidades jesuitas (y también algunas otras) jugaron un papel fundamental en la profundización y la divulgación de la cultura humanista, a partir de la primera parte de la Edad Moderna. ${ }^{24}$

El sustantivo "Humanismo" que fue fomentado en las universidades jesuitas pasó a ser acompañado, más tarde, por el apelativo de "cristiano". Pero, al menos hasta la llustración, puede llegar a afirmarse que el Humanismo se identificaba plenamente con el pensamiento cristiano. ${ }^{25}$

Regresemos a los discursos de Peter Hans Kolvenbach (2008, p. 253), por ejemplo al que pronunció en las facultades universitarias de Notre Dame de la Paix y en el que recordaba que esta dimensión era un elemento central de la pedagogía jesuita:

Cfr. 12 "La educación jesuítica exalta toda la gama de capacidades y de logros intelectuales de la persona humana".

En realidad, en Kolvenbach, la "humanitas" incorpora un matiz muy relevante vinculado con la forma- ción intelectual y racional de la persona, que por supuesto no es ajeno a la mejor tradición de la cultura humanista y de las universidades jesuitas. Más aún, el hoy rector de la Universidad de Deusto José María Guibert (2009, pp. 168-170) incidía en tal interpretación de esta forma: "Es mejor utilizar el término formación intelectual para referirnos a esta dimensión, la que destaca de manera explícita el valor de la razón, la mente o el intelecto humanos, o dicho de otra manera, las competencias cognitivas".

\section{LA “FIDES”, CARÁCTER CONFESIONAL DE LAS UNIVER- SIDADES JESUITAS}

Ante la sociedad civil, la "fides" representa el elemento más distintivo de la Compañía de Jesús, como orden religiosa que es; amén de que configura también su "razón de ser" y la de cada una de sus obras, incluida por supuesto las universidades. La "fides" define el carácter confesional de las universidades jesuitas. Las universidades no están meramente inspiradas en el Humanismo cristiano; sino que forman parte del organigrama de la Iglesia Católica y, de principio a fin, de su proyecto apostólico.

En 2001 y en Roma, en la conferencia que pronunció ante universidades jesuitas de todo el mundo, Kolvenbach (2008, p. 196) ya advertía de la necesidad de repensar qué implicaciones traía consigo el carácter confesional de las universidades jesuitas:

Cfr. 14 "En un mundo en que en unas regiones la secularización y la descristianización ganan cada vez más terreno, mientras en otras el cristianismo es prácticamente irrelevante, el tema de la identidad de nuestras universidades y de la visibilidad de tal identidad ha saltado a primer plano".

Lo cierto es que en un mundo secularizado la variable "fides" puede ser interpretada, cada vez más, como conflicto. Con todo, es bueno evocar que, en la Baja Edad Media, algunas de las primeras y más brillantes universidades, como las de Bolonia o París, habían surgido como resultado de conflictos, muchas veces de carácter eclesial (Tamayo y Salmorán, $1987 / 2005$, pp. $45-81$ ) y que el origen de los centros de estudios superiores jesuitas también se enmarcó en el seno de disputas de esta naturaleza. En este contexto, la fundación y la reforma de las universidades católicas o el cultivo del apostolado intelectual se van a contar entre las propuestas del Concilio de Trento; convirtiéndose los jesuitas en protagonistas y su Ratio Studiorum en guía, a lo largo y ancho de Europa (Borrero Cabal, 2008, pp. 438-440). 


\section{LA "IUSTITIA" COMO ELEMENTO ESPECÍFICO DE LAS UNIVERSIDADES JESUITAS}

Naturalmente la dimensión de "iustitia"n6 es recurrente, una y otra vez, en el conjunto de los discursos de Kolvenbach; pero presta particular atención a esta variable en algunos de ellos. En concreto, el discurso en la Universidad de Santa Clara de 2000, en California, ante los representantes de alrededor de treinta centros de estudios superiores de la Compañía de Jesús de Estados Unidos, se considera el más focalizado en la promoción de la justicia. Aunque, por el contexto geográfico en el que tenía lugar, este discurso era también más dirigido a las universidades de los países desarrollados.

Esta conferencia llevaba por título el mismo que el Decreto 4 de la Congregación General XXXII: Servicio de la fe y promoción de la justicia. De hecho, el discurso se pronunció con ocasión de los 25 años de la celebración de la citada Congregación General. Kolvenbach, presente como delegado en la Congregación General XXXII, mostraba así su plena sintonía con este importante decreto $y$, sobre todo, con la potencial conversión que podía suponer para la Compañía de Jesús, en sus apostolados educativo e intelectual.

Cuando Kolvenbach, en cualquiera de sus discursos universitarios, se refiere -de modo explícito o implícito- a la dimensión de "iustitia", sintoniza claramente con la dialéctica y el pensamiento de su predecesor y carismático Arrupe, ${ }^{27}$ con los principios del Decreto 4 de la Congregación General XXXII y, lógicamente también, con los de las dos posteriores Congregaciones Generales que el presidirá.

Es indiscutible que la Compañía de Jesús dio un salto cualitativo en la propia semántica, para confirmar, diferenciar y hacer que su compromiso, por un cambio social de envergadura y de carácter estructural, ganara en identidad. Transcurridas varias décadas desde la celebración de la Congregación General XXXII, es fácil comprobar que este lenguaje sigue siendo inusual cuando otras universidades (católicas o civiles) abordan su compromiso social. Asimismo, la dialéctica de la Responsabilidad Social Universitaria (RSU) pocas veces hace uso de este lenguaje.

¿Por qué postulaba Peter Hans Kolvenbach (2008, pp. 181-186) para orientar la promoción de la justicia desde los centros de estudios superiores jesuitas? Volviendo al discurso en la Universidad de Santa Clara, quien fuera prepósito general de la Compañía de Jesús entre 1983 y 2008, venía a proponer lo siguiente:

- Evaluar la misión universitaria de acuerdo a lo que los alumnos lleguen a ser o según actúen en el futuro. Las iniciativas que los alumnos desarrollen en el marco universitario, a favor de los demás y de su entorno social, son esencialmente "actividades para su formación". Porque en su itinerario formativo, lo que los alumnos requieren "es un compromiso cercano con el pobre y el marginado, para aprender de la realidad y llegar a ser un adulto en solidaridad". De ahí que "el auténtico criterio para evaluar las universidades de la Compañía no es lo que nuestros estudiantes hagan sino lo que acaben siendo y la responsabilidad cristiana adulta con la cual trabajen en el futuro a favor de sus prójimos y de su mundo".

- Desarrollar una oferta de formación para el servicio total. Considerando que la formación total ha conformado uno de los rasgos diferenciales de la pedagogía jesuita, "la persona completa del mañana debe tener (...) una solidaridad bien informada". Y esto supone imprescindiblemente llegar a poseer "una conciencia instruida de la sociedad y de la cultura, con la que contribuir generosamente en el mundo tal cual es". La misión es conseguir que cada alumno se convierta en "una persona completa y solidaria para tomar sobre sí la responsabilidad del mundo real". Esto implica, más que asimilar conocimientos teóricos, alguna "experiencia directa" que toque "al corazón", ya que así "la mente se puede sentir desafiada a cambiar".

- Incentivar una actitud crítica ante el mundo. También de acuerdo con la tradición pedagógica de la Compañía de Jesús, se establece que los alumnos "tienen que dejar entrar en sus vidas la realidad perturbadora de este mundo, de tal manera que aprendan a sentirlo, a pensarlo críticamente, a responder a sus sufrimientos y a comprometerse con él de forma constructiva".

- Ubicar las iniciativas de servicio a la comunidad y a los colectivos desfavorecidos en el núcleo de la actividad educativa. Las acciones de esta naturaleza han de pasar de ser "algo opcional o periférico" a formar parte del "núcleo mismo del programa de estudios de toda universidad de la Compañía".

- Acercar las disciplinas y las líneas de investigación a la realidad y al cambio social. Es necesario ir más allá de "los cánones de cada disciplina" para "adentrarse en lo más profundo de 
la realidad humana, para ayudar a hacer del mundo un lugar más habitable". Cada "disciplina, más allá de su necesaria especialización, tiene que comprometerse de forma adecuada con la sociedad, con la vida humana, con el ambiente, teniendo siempre como preocupación moral de fondo cómo deberían ser los hombres para poder vivir juntos". Para ello, es también necesario "una colaboración orgánica con aquellos que, en la Iglesia y en la sociedad, trabajan entre los pobres y en favor de ellos, buscando activamente la justicia".

\section{¿FORMACIÓN DE CLASES DIRIGENTES Y PROMOCIÓN DE LA JUSTICIA SOCIAL?}

Sin duda, el animar la formación de líderes ha conformado uno de los elementos más distintivos de los objetivos pedagógicos de la Compañía de Jesús. Pero, no rara vez, ha supuesto también uno de los aspectos más controvertidos; en la medida que los jesuitas han sido -por ello- acusados de connivencia y hasta de complicidad con las clases más poderosas, y sobre todo cuando han favorecido a una elite económica y social determinada. ${ }^{28}$

La razón esencial de formar líderes ha estribado en el deseo de conseguir un "efecto multiplicador", es decir, un impacto social de más envergadura. La búsqueda de este efecto fue también la razón por la cual los jesuitas ejercieron, a menudo, como confesores de las clases dirigentes, incluso era común que lo fueran de distintas familias de la Realeza. ${ }^{29}$

En suma, y a diferencia de otras órdenes o congregaciones religiosas que también se crearon en la Edad Moderna, como por ejemplo las Escuelas Pías, la mayor parte del alumnado de los colegios y de las universidades jesuitas pertenecía -desde un inicio- a las clases sociales privilegiadas; es decir, a la burguesía, a la nobleza y hasta a la aristocracia. Ellas fueron muchas veces quienes solicitaron a los jesuitas la fundación de nuevos centros educativos y quienes, además, les proveyeron de los recursos económicos necesarios para este fin.

Así y todo, es justo aclarar que Ignacio de Loyola mostró su voluntad e interés para que la fundación de los colegios contara con los recursos necesarios con el fin de garantizar que se incorporaran todo tipo de estudiantes en ellos, también los más pobres; a la vez que solicitaba al profesorado que atendiera de modo especial las necesidades de los alumnos pertenecientes a las clases desposeídas (Características de la educación de la Compañía de Jesús, p. 28). E Ignacio de Loyola es- taba en el convencimiento de que la formación de las clases dirigentes, para ser efectiva, no podía descuidar la capacitación del resto de la sociedad. ${ }^{30}$

Más aún, hay quien afirma con contundencia que "todos los Colegios de la antigua Compañía, siglos XVI al XVIII inclusive, eran externados gratuitos" (Fernández en Kolvenbach, 1990, p. 8). Por lo tanto el predominio de las clases más privilegiadas en buen número de las aulas jesuitas, al menos hasta los años de la disolución de la orden, habría que atribuirse esencialmente a otras razones que no son económicas. ¿Cuáles podrían ser los motivos? Principalmente tendríamos que pensar en los siguientes:

- Los criterios, por los que los jesuitas finalmente fueron optando, para la divulgación de su oferta educativa y para la selección de los alumnos.

- La poca sensibilización de la sociedad en general para promover la capacitación de las clases populares.

- La ubicación geográfica de los centros educativos en zonas frecuentadas por clases sociales más pudientes.

- $\quad \ldots$

Es cierto que, a caballo de los siglos XVII y XVIII, los jesuitas ya contaban con un amplio número de notorios y prestigiosos centros de enseñanza en Europa o en algunas florecientes urbes de América Latina, y en los que principalmente las clases sociales acomodadas eran educadas. Pero hay también que constatar que, por ejemplo en las Reducciones de la selva de Paraguay, crearon -en esa misma época- las primeras escuelas para las poblaciones indígenas, que huían de la esclavitud.

No obstante, siguiendo la tradición realista de la pedagogía de la Compañía de Jesús, un jesuita involucrado en esta misión aclaraba que "el cambio (...) en la dirección fe - justicia no provendrá fundamentalmente del hecho de admitir más pobres (...) (hay limitaciones muy evidentes para ello), sino de la trasformación que puedan vivir en actitudes y valores nuestros alumnos y alumnas, nuestros profesores" (Fernández Dávalos, 2005, p. 13).

\section{PALABRAS FINALES}

Ya para ir concluyendo, aunque los discursos de Kolvenbach hayan sido pronunciados en universidades localizadas en puntos muy diversos; con coyunturas sociales, culturales, políticas, educativas y religiosas extraordinariamente distintas, los contenidos -referidos al paradigma que ahora estamos estudiando- no varían de modo sustancial. 
En líneas generales, cabe decir que el término "utilitas" puede llegar a ser transversal al conjunto de las universidades, pero circunscribiéndose solo a este ámbito se corre el peligro de desembocar en que la universidad quede simplificada y carcomida por el puro "profesionalismo", la "tecnocracia" o el "utilitarismo". La dimensión "humanitas", que es también tenida en consideración -de un modo u otro- en muchas universidades no jesuitas, ha pretendido poner remedio a este riesgo. La perspectiva "fides" es un elemento identitario de toda universidad confesional, es decir, no solo de las que pertenecen al orbe católico o incluso cristiano; sino de las que están asimismo presentes, de modo particular, en tierra del Islam. Y, por último, la incorporación de la variable "iustitia" -al mismo nivel que las otras tres dimensiones- comprende, como hemos ya escrito en páginas atrás, un punto diferenciador de las universidades de la Compañía de Jesús.
Dicho de otro modo, si las variables "utilitas" y "humanitas" pueden ser asimiladas por el conjunto de las universidades, las dimensiones "fides" e "iustitia" -servicio de la fe y promoción de la justicia- son las peculiaridades que, desde el paradigma de Ledesma - Kolvenbach, quieren distinguir a las universidades jesuitas, como en realidad al resto de obras apostólicas de la Compañía de Jesús. Así y todo, y como hemos visto, el modelo de Ledesma - Kolvenbach no está constituido por cuatro variables estancas; sino por cuatro dimensiones que no son un "juego de suma cero", sino que se retroalimentan, se enriquecen y se interrelacionan con otros espacios connaturales o propios de la actividad universitaria.

Está claro asimismo que el paradigma de Ledesma Kolvenbach queda todavía en fase de desarrollo o deba ser interpretado como un punto de partida; aunque ya no corresponda, a quien fuera el inmediato sucesor de Arrupe, el seguir cavilándolo, matizándolo, concretándolo y liderando su implantación.

\section{NOTAS}

1 Ignacio de Loyola acudió a la universidad parisina a pie, procedente de la península Ibérica. Cuando se acercaba a los 40 años, no había logrado todavía ningún título académico, había optado por entregarse a la absoluta pobreza y sobrevivía de las limosnas. Se inscribió en la universidad parisina como Ignatius, nombre por el cual iba a ser conocido a partir de entonces; abandonando para siempre el de Iñigo, con el que fue bautizado.

Quienes pronunciaron sus primeros votos como jesuitas, en la colina parisina de Montmartre y en el año 1534, fueron -además de Ignacio de Loyola- seis jóvenes universitarios procedentes de lugares diversos, como Castilla, Portugal, Saboya y Navarra. "De los estudiantes tienen todo el entusiasmo, la exhuberancia de la vida, el sentido de la camaradería y el gusto por el riesgo" (Ravier, 1991, p. 82).

2 En la fórmula de la fundación de la Compañía de Jesús, recogida en 1540 en la bula Regimini militantis Ecclesiae del Papa Pablo III, no se hizo mención expresa a los apostolados propiamente educativo o intelectual, ya que restringía la enseñanza al apartado catequético.

3 En 1541, en la primera redacción de las Constituciones de los jesuitas, se dejó explícito el deseo de "no estudios ni lecciones en la Compañía". Esto ponía de manifiesto la intención de los jesuitas por no crear centros formativos ni para la capacitación de sus propios miembros ni para otro tipo de personas. En un principio, la Compañía de Jesús sí se comprometió en fundar colegios como residencias de estudiantes jesuitas, pero insistimos en que sin interés de servir como centros de enseñanza. Sin embargo, la oferta formativa a la que los jesuitas estudiantes podían acceder no cumplía con las expectativas de la Compañía de Jesús. De modo que la orden empezó a hacerse cargo de la formación de sus propios miembros en estos colegios-residencias, y pronto fue abriendo por extensión sus aulas al resto de los jóvenes (Batllori en Delgado Criado, 1994, pp. 57-58).

4 Para conocer la evolución de la Compañía de Jesús, en las decisivas décadas de los 60 y 70, véanse por ejemplo Bangert (1981) y Woodrow (1985). Y, desde una perspectiva más crítica, consúltese De la Cierva (1986).

5 Es importante insistir en que los jesuitas tomaron como referencia el discernimiento que el conjunto de la Iglesia Católica estaba llevando a cabo en el plano de la acción por la justicia social. Véase, sobre este asunto, por ejemplo, La justicia en el mundo. Nuevas responsabilidades de la Iglesia en el campo de la justicia.

6 En palabras del jesuita José María Margenat (2008, p. 653), la apuesta por el trabajo por la justicia conduce también a sustituir "el arte de la prudencia" arraigado en la cultura educativa de la Compañía de Jesús:

"En la tradición educativa de los jesuitas el arte de la prudencia parece que jugó un papel importante (...), ciertamente un papel mayor que el del arte de la justicia. Hoy no podemos ya, ciertamente, conformarnos con la prudencia (...), sino apostar por la justicia y por el reconocimiento de los derechos de los otros en la comunidad".

7 El Decreto 4 de la Congregación General XXXII ha sido también calificado como "Decreto 40"; habida cuenta de la trascendencia que ha supuesto para la trayectoria de la Compañía de Jesús, durante las últimas décadas. Para un análisis exhaustivo véase, por ejemplo, Ivern (1976). 
8 No podemos olvidar tampoco las consecuencias trágicas del compromiso a favor de la justicia social desde las universidades jesuitas. El rector Ignacio Ellacuría y el resto de jesuitas que residían en el "campus" de la Universidad Centroamericana "José Simeón Cañas" fueron asesinados en 1989 a causa de su denuncia de las injusticias. Ellacuría era una personalidad de gran capacidad intelectual, que realizó importantes disertaciones sobre la misión de la universidad a favor de los colectivos sociales más desfavorecidos. A este respecto, véase Ellacuría (1999).

9 Con todo, el jesuita Agustín Udías (2014, p. 319) anotaba que, desde la Congregación General XXXII, se ha producido una desatención a las ciencias en la Compañía de Jesús: "El énfasis puesto en la Congregación General 32 en la promoción de la fe y la justicia no pretendía disminuir la dedicación de los jesuitas a la ciencia, pero de hecho contribuyó a ello (...). Los superiores empezaron a ver que la dedicación a las ciencias naturales no es una necesidad urgente en el trabajo de los jesuitas hoy".

10 No se cuestiona tampoco que la justicia, tal y como los evangelios hablan de ella, queda vinculada en buena parte al compromiso y con la mejora de las condiciones de vida de los colectivos más desfavorecidos o con la desaparición de las desigualdades sociales. Esta interpretación está también presente a menudo en el judaísmo y en la tradición veterotestamentaria. El relato de las "Bienaventuranzas" o el "Sermón de la Montaña", contenido en dos evangelios sinópticos (Mateo y Lucas) y considerado asimismo como uno de los pasajes más relevantes del Nuevo Testamento o incluso como la síntesis del mensaje de Jesús de Nazareth, deja especialmente constancia de la opción preferencial del cristianismo por los grupos sociales más indefensos o vulnerables. Acudiendo a la versión del evangelio de Mateo (5, 3-12), el cristianismo toma partido -por ejemplo- por los siguientes grupos, que pueden ser descubiertos en cualquier tipo de sociedad o contexto histórico: "los pobres de espíritu", "los que lloran", "los que tienen hambre y sed de justicia", "los que padecen persecución por causa de la justicia".

11 Ignacio de Loyola ya aconsejaba a los jesuitas cómo actuar en la denuncia de injusticias, como por ejemplo hizo en la carta " $a$ los padres enviados a Trento", en 1546: "Si las cosas de que se hablare son tan justas, que no se pueda o deba callar, dando allí su parecer con la mayor quietud y humildad posible, concluyendo salvo otro parecer mejor" (Iparaguirre, 1963, p. 669). Esta carta es una de las más sugerentes para identificar, asimismo, lo que se conoce como "liderazgo ignaciano".

12 Este artículo ha sido elaborado a partir de la tesis doctoral, defendida en 2013, en la Universidad de Deusto, y bajo el título La educación superior de la Compañía de Jesús y la promoción de la justicia. Formulación de propuestas, selección de buenas prácticas y análisis de la actitud del personal adscrito a las universidades jesuitas de España. La tesis fue dirigida por José María Guibert, jesuita y actual rector de la Universidad de Deusto.

13 Es bueno recordar que el jesuita Luigi Taparelli fue quien acuñó y definió, por primera vez, el término "justicia social", antes de llegar a la mitad del siglo XIX. Taparelli, nacido en Turín en 1793 y fallecido en Roma en 1862, fue uno de los fundadores de la conocida revista Civiltà Cattolica, promovida por la Compañía de Jesús. Ha sido, asimismo, uno de los precursores de la doctrina social de la Iglesia Católica. Taparelli abrió un camino, en el campo de la filosofía social de la Iglesia Católica, que no solo nunca se ha cerrado sino que, además, ha ido ganando más entidad y desarrollo con el transcurso de las décadas. En su obra Ensa- yo teórico de derecho natural apoyado en los hechos, Taparelli (1866/1884, p. 173) afirmaba lo siguiente:

"La justicia social es para nosotros justicia entre hombre y hombre (...). Porque un hombre y otro hombre no son sino dos veces la humanidad (...). De donde tengo que concluir que la justicia social debe igualar de hecho a todos los hombres en lo tocante a los derechos de humanidad, como el Criador los hizo iguales en naturaleza; y que el hombre que obra tomando por norma la justicia, llena las intenciones de quien le crió".

14 Peter Hans Kolvenbach nació cerca de Nimega (Holanda) en 1928 e ingresó en el noviciado de la Compañía de Jesús en 1948. Fue destinado a Líbano en 1958. En 1981, fue designado rector del Pontificio Instituto Oriental de Roma. Dos años después, sustituyó a Pedro Arrupe al frente de la Compañía de Jesús.

15 Esto no quiere decir, ni mucho menos, que las instituciones educativas no universitarias -regentadas por los jesuitas- no cuenten con orientaciones actualizadas que les guíen en su misión. A modo de ejemplo, véase Gil Coria y Labrador (1999).

16 El concepto de identidad y misión es lugar común para reflexionar sobre los valores y la cultura organizacional en las obras apostólicas de los jesuitas. El vicecanciller de la Universidad de Deusto, el jesuita Javier López Ariztegui (2014), reflexionaba con estas palabras sobre su significado y alcance:

"Nuestra identidad, como se puede comprobar, es algo dinámico y profundo. Se nutre de un espíritu original, toma cuerpo, interacciona, crece moldeada por el tiempo presente y es atraída desde el futuro".

17 Siendo rector de la Universidad de Deusto, y como crítica velada al "utilitarismo", el jesuita Jaime Oraá hablaba así: "Universidades y los intelectuales por profesión, podemos tener la tendencia de vivir excesivamente aislados y alejados del mundo real, de la injusticia nacional e internacional"' (Oraá, 2007, p. 212).

18 En particular, las universidades jesuitas de América Latina son las que más están trabajando expresamente desde la Responsabilidad Social Universitaria (RSU). Véase, al hilo de esto, por ejemplo, Informe final del proceso de autoevaluación de la responsabilidad social universitaria en AUSJAL.

19 La Ratio Studiorum de 1599 constituyó una de las grandes aportaciones de la Compañía de Jesús a la historia de la pedagogía. Fue aprobada siendo superior general Claudio Acquaviva y contribuyó de manera notable al desarrollo del apostolado educativo de los jesuitas.

Los colegios eran 372 a la muerte del general Claudio Acquaviva (1615) y, un siglo después, sumaban 610. Cuando la Compañía de Jesús fue disuelta, a finales del siglo XVIII, eran 621 (O’Neill y Domínguez, 2001, p. 683).

La Ratio Studiorum de 1599 fue redactada -a lo largo de los años- a partir del intercambio, la búsqueda de sinergias o el descubrimiento de los mejores métodos, que emanaban de las experiencias educativas de los incipientes centros de enseñanza impulsados por la Compañía de Jesús. La Ratio Studiorum de 1599 fue fruto de un trabajo en común, de compartir un mismo proyecto educativo o de formar parte de una misma misión; lo cual ya fue por sí solo un logro que tampoco encontraba precedentes.

La implementación del paradigma de Ledesma - Kolvenbach, y exactamente la determinación de pautas concretas que orienten su aplicación en las universidades jesuitas, requerirá también el mismo esfuerzo de contraste y trabajo común. 
20 Utilizaremos la nomenclatura latina de las cuatro dimensiones del paradigma, al ser ya común en las universidades jesuitas. Si bien este jesuita no estableció estos latinismos, hasta su último discurso académico que tuvo lugar en la Universidad Gregoriana de Roma, en 2007 (Kolvenbach, 2008, pp. 259263). Por ejemplo poco antes, en 2006 , en su discurso en las facultades universitarias de Notre Dame de la Paix, Kolvenbach empleó otros cuatro términos para sintetizar su propuesta: "Práctica", "social", "intelectual" y "religiosa" (Kolvenbach, 2008, pp. 250-255).

21 Dicho de otra manera, esto significa "ayudar al desarrollo más completo posible de todos los talentos dados por Dios a cada persona individual como miembro de la comunidad humana" (Características de la educación de la Compañía de Jesús, p. 14).

22 Recientemente el Secretariado para la Justicia Social y la Ecología de la Compañía de Jesús (Álvarez, 2014) ha publicado un documento bajo el título de La promoción de la justicia en las Universidades de la Compañía, con la participación de jesuitas de diferentes continentes. El texto enfatiza en las disfunciones sociales que el "utilitarismo" puede acarrear, al tiempo que propone a la educación superior de la orden de Ignacio de Loyola como alternativa:

"La educación universitaria habitual suele limitarse a esta motivación de utilidad, proporcionando las herramientas intelectuales para un desempeño profesional distinguido. El riesgo reside en que un sentido meramente utilitario de la educación conduzca al desprecio o a la subordinación de los valores precisos para la construcción de una sociedad justa. A las universidades jesuitas no les basta únicamente con transmitir una racionalidad instrumental. Cuando esto sucede de forma exclusiva, la educación deriva fácilmente hacia la injusticia y la exclusión, pues acumula saberes, haberes y poderes para los mejor situados" (pp. 9-10).

23 Y esta tradición ha de continuar vigente en la Compañía de Jesús, según el sucesor de Kolvenbach: "La Ética, las Humanidades y las Ciencias Sociales deberán tener mayor protagonismo en el diseño del modelo de sociedad para el siglo XXI, si no queremos estar supeditados al dictado de la economía y el mercado, con el consiguiente empobrecimiento moral y la creación de abismos cada vez mayores entre los que tienen y los que no tienen" ( $\mathrm{Ni}$ colás, Lección inaugural del curso académico 2011-2012 en la Universidad de Deusto, p. 3).

24 Dentro de las humanidades, en los albores de la Compañía de Jesús, habría también que incluir el cultivo de lo que hoy denominamos ciencias exactas. Por ejemplo, en la Ratio Studiorum de 1599 se recogían ya algunas normas, dirigidas a los profesores de matemáticas y destinadas a servirles de orientación para sus clases.

\section{BIBLIOGRAFÍA}

Agúndez Agúndez, M. (2008). El paradigma universitario Ledesma - Kolvenbach. Revista de Fomento Social, 252, 63, pp. 603-631.

Álvarez, P. (ed.) (2014). La promoción de la justicia en las Universidades de la Compañía. Promotio lustitiae, 116, 2014/3.

Bangert, W. V. (1981). Historia de la Compañía de Jesús. Santander: Sal Terrae.

Benedicto XVI (2011). Discurso en el encuentro con profesores universitarios jóvenes. En Encuentros con la comunidad universitaria.
En este contexto histórico, debemos de recordar la figura de Cristóbal Clavius, jesuita del Colegio Romano, que impulsó de manera decisiva el estudio de las ciencias y, en concreto, de las matemáticas y la astronomía. Clavius fue, igualmente, el precursor del calendario Gregoriano, vigente desde finales del siglo XVI. Precisamente, uno de los cráteres más grandes de la Luna lleva su nombre. Véase, por ejemplo, Guibert (2012).

25 Sin embargo, el jesuita Ignacio Cacho (2004) prefería matizar que lo que distingue al Humanismo de Ignacio de Loyola era el "humanismo jesuocéntrico".

26 Bien sabemos que el concepto de justicia ha sido recurrente una y otra vez en la historia del pensamiento. Un documento atribuido a Anaximandro de Mileto es el más temprano en el que se medita sobre la acepción de justicia; vinculándola tanto al orden natural de las cosas como a la buena organización de la comunidad humana. Anaximandro de Mileto vivió entre los siglos VII y VI antes de Cristo (Martínez Navarro en Cortina, 2000, pp. 163-164).

27 Los discursos propiamente universitarios de Pedro Arrupe no ganaron tanta notoriedad como los que han alcanzado los de Kolvenbach. Sin embargo, alguna de las cartas de Arrupe o de sus discursos educativos han gozado de gran influencia en la Compañía de Jesús. Entre ellos se pueden citar la Carta a los antiguos alumnos de la Compañía de Jesús y el Discurso al $X$ Congreso de la Confederación Europea de Antiguos Alumnos de Jesuitas, ambos inéditos.

28 Aunque debemos anotar, como también recordaba Arrupe, que el superior general de la Compañía de Jesús, Jean - Baptiste Janssens, ya mostraba su preocupación por la excesiva presencia de las elites económicas en los centros educativos jesuitas; tal y como por ejemplo señaló en 1949: "Hemos de preguntarnos si nuestros alumnos y conocidos no han recibido de nosotros confirmación de los prejuicios de clase que acaso traían de sus familias" (Así lo indica Torelló en La evolución de los colegios de la Compañía según las directrices del Padre Arrupe [documento inédito]).

29 Véase, por ejemplo, Lamet (2004).

30 "Seguramente, el fermento de la sociedad que él tenía a la vista requería unos pocos líderes de primera magnitud y bien formados; pero necesitaba también un gran número de seguidores formados para una visión semejante. Sin la ayuda de estos seguidores en gran número era poco verosímil que los líderes lograran puestos en los que pudieran desplegar su influencia o desempeñar su papel con éxito. La levadura no fermentaría la masa; sino que la masa sofocaría a la levadura" (Ganss, 1958, p. 206).

Instituto de Antropología y Ética. Universidad de Navarra. Documentos del Instituto de Antropología y Ética, 16, pp. 5-7. Disponible en https://www.unav.edu/documents/8871060/8964433/16-Enc uentros+con+la+comunidad+universitaria.pdf/8a782ad3-a9074a88-a2cb-c66c873fef18

Borrero Cabal, A. (2008). La universidad. Estudios sobre sus orígenes, dinámicas y tendencias (tomo I). Bogotá: Pontificia Universidad Javeriana. 
Cacho, I. (2004). Dos Humanismos del Renacimiento. Anuario del Instituto Ignacio de Loyola, 11, pp. 75-124.

Cava Mesa, M. J. (2007). Pedro Arrupe. Jesuita y bilbaino universal. Bilbao: Muelle de Uribitarte Editores.

Cortina, A. (dir.). (2000). 10 palabras clave en ética (3a ed.). Pamplona: Verbo Divino.

De la Cierva, R. (1986). Jesuitas, Iglesia y marxismo 1965-1985. La teología de la Liberación desenmascarada. Barcelona: Plaza \& Janes.

Delgado Criado, B. (coord.) (1994). Historia de la educación en España y América. La educación en la España Moderna (siglos XVI - XVIII). Madrid: Morata. Fundación Santa María.

Ellacuría, I. (1999). Escritos universitarios. San Salvador: UCA Editores.

Farrell, A. P. (1970). The Jesuit Ratio Studiorum of 1599. Conference of Major Superiors of Jesuits. [En línea]. Disponible en http:// www.bc.edu/sites/libraries/ratio/ratio1599.pdf

Fernández Dávalos, D. (2005). La universidad de la Compañía de Jesús: su identidad y espiritualidad. (Elementos para su construcción). Seminario AUSJAL sobre Identidad, Espiritualidad y Universidad. [En línea]. Disponible en http://www.ausjal.org/ tl_files/ausjal/images/contenido/Documentos/Publicaciones/ Educacion\%20superior/La\%20Universidad\%20de\%20la\%20Compania\%20de\%20Jesus\%20su\%20identidad\%20y\%20espiritualidad\%20-\%20David\%20Fernandez\%20Davalos\%20S.J\%20_1_.pdf

Ganss, J. E. (1958). Universidad y educación jesuíticas ideadas por S. Ignacio. Estudio histórico de la educación católica. Ciudad TrujiIlo - La Habana - San Juan de Puerto Rico: Ediciones y Publicaciones Antillenses.

Gil Coria, E. y Labrador, C. (eds.). (1999). La pedagogía de los jesuitas, ayer y hoy. Madrid: Universidad Pontificia Comillas.

Guibert, J. M. (2009). La incidencia de las universidades de la Compañía de Jesús en la cultura europea. Gregorianum, 90, 1, pp. 159-176.

Guibert, J. M. (2012). Cristóbal Clavius. Jesuitas, 112, pp. 16-17.

Hortal Alonso, A. (2008). Utilitas: la dimensión práctica de la formación universitaria. Revista de Fomento Social, 63, 252, pp. 633-650.

Iparaguirre, I. (ed.) (1963). Obras de San Ignacio de Loyola (2a ed.). Madrid: Biblioteca de Autores Cristianos.

Ivern, F. (1976). Nuestra misión hoy: El servicio de la fe y la promoción de la justicia. Su significado y consecuencias a la luz de nuestro compromiso social actual. En Identidad y misión de la Compañía de Jesús hoy. Semana Nacional de Loyola. 1-6 Setiembre 1975. Bilbao: Mensajero, pp. 49-77.

Kolvenbach, P. H. (1990). La Universidad: espacio para la unidad de las ciencias. Orientaciones Universitarias, 4, pp. 5-24.

Kolvenbach, P. H. (2008). Discursos universitarios. Madrid: UNIJES.

Lamet, P. M. (2004). Yo te absuelvo, majestad: confesores de reyes y reinas de España. Madrid: Temas de Hoy.

López Ariztegui, J. (2014). Reflexiones sobre nuestra identidad y misión. Revista Deusto, 124, p. 19.

Margenat, J. M. (2008). Iustitia: educar para una ciudadanía responsable. Revista de Fomento Social, 63, 252, pp. 651-669.
O’Neill, C. E. y Domínguez, J. M. (dirs.). (2001). Diccionario histórico de la Compañía de Jesús. Biográfico-Temático (tomo I). Madrid - Roma: Universidad Pontificia Comillas.

Oraá, J. (2007). Algunas reflexiones acerca del papel de las universidades jesuitas en la promoción de la justicia. En AA.VV. Jesuitas: una misión, un proyecto. Bilbao: Universidad de Deusto, pp. 203-221. Disponible en http://www.deusto-publicaciones.es/ deusto/pdfs/forum/forum19.pdf

Plazaola, J. (1999). Por qué y cómo la Compañía de Jesús vino a ser una Orden educadora. Anuario del Instituto Ignacio de Loyola, 6, pp. 7-25.

Ravier, A. (1991). Ignacio de Loyola. Fundador de la Compañía de Jesús. Madrid: Espasa - Calpe.

Sánchez Marco, F. El ministerio intelectual de la Compañía y la nueva evangelización. En Guibert, J. M. (coord.) (2010). Cooperativismo, empresa y universidad. In memoriam de Dionisio Aranzadi Tellería SJ. Bilbao: Universidad de Deusto, pp. 453-476.

Tamayo y Salmorán, R. (1987/2005). La universidad, epopeya medieval. Notas para un estudio sobre el surgimiento de la universidad en el alto medievo (3a ed.). México: Universidad Nacional Autónoma de México. Unión de Universidades de América Latina.

Taparelli, L. (1866/1884). Ensayo teórico de derecho natural apoyado en los hechos (tomo I) (2a ed.). Madrid: Nueva Librería e Imprenta de San José.

Udías, A. (2014). Los jesuitas y la ciencia. Una tradición en la Iglesia. Bilbao: Mensajero.

Woodrow, A. (1985). Los jesuitas. Historia de un dramático conflicto. Barcelona: Planeta.

\section{Recursos de Internet}

Características de la educación de la Compañía de Jesús. [En línea]. Disponible en http://www.flacsi.net/wp-content/uploads/2011/11/ Caracteristicas-de-la-Educacion-de-la-S.J.-1986.pdf

Decreto 4, Nuestra misión hoy: Servicio de la fe y promoción de la justicia. [En línea]. Disponible en http://www.sjweb.info/sjs/documents/CG32_D4_esp.pdf

Informe final del proceso de autoevaluación de la responsabilidad social universitaria en AUSJAL. [En línea]. Disponible en http:// www.ausjal.org/tl_files/ausjal/images/contenido/Documentos/Publicaciones/Investigaciones\%20Publicadas/InformeFinalRSU2011.pdf

La justicia en el mundo. Nuevas responsabilidades de la Iglesia en el campo de la justicia. Sínodo Mundial de Obispos, Roma 1971. [En línea]. Disponible en http://www.confer.es/508/activos/texto/wcnfr_pdf_2566-U1bgv1kNzDHk7cr2.pdf

Lección inaugural del curso académico 2011-2012 en la Universidad de Deusto, pronunciada por Adolfo Nicolás S.J, Gran Canciller de la Universidad y Superior General de la Compañía de Jesús, con motivo del 125 aniversario de la Universidad de Deusto. Bilbao, 9 de septiembre de 2011. [En línea]. Disponible en http:// www.ausjal.org/tl files/ausjal/images/contenido/Documentos/Publicaciones/Identidad\%20y\%20Mision/Leccion\%20Inaugural\%20\%20Deusto\%20curso\%202011-2012\%20P.Adolfo\%20 Nicolas.pdf 